\section{MICROBIOME}

\section{A microbiome tool for deriving the universal blood donor}

Nat. Microbiol. https://doi.org/10.1038/s41564019-0469-7 (2019)

An enzyme found in a bacterium in the human gut microbiome is able to convert blood type A into the universal donor blood type $\mathrm{O}$.

Red blood cells are defined on the basis of the carbohydrate antigens on their cell surface. Blood type A contains antibodies against blood type $B$, and vice versa. There may be lethal consequences if the wrong blood type is donated. Blood type $\mathrm{O}$ has none of these antigens and can be transfused between individuals with the same rhesus type. A large supply of this type of blood is needed for emergencies.

A group of scientists in Canada hypothesized that the gut microbiome might contain enzymes that metabolize the blood antigens since these antigens are found on the gut wall. Through screening they found a bacterium that was able to metabolize blood group A to blood group $\mathrm{O}$. This has the potential to alter the future blood supply. HS

https://doi.org/10.1038/s41591-019-0529-9

\section{IMMUNOTHERAPY}

\section{Testing for repeat adverse events}

JAMA Oncol. https://doi.org/10.1001/

jamaoncol.2019.1022 (2019)

Immune checkpoint blockade for cancer therapy can result in severe immune-related adverse events, and restarting this therapy after cessation can induce similar events, but the risk-reward ratio suggests that it is worth the risk.

Immune checkpoint blockade is based on reactivating a patient's immune system to target their tumor. This can lead to severe adverse immune events as a result of immune system activation. While most of these events resolve after they arise, it is unknown if it is safe to readminister immune checkpoint blockade after therapy is stopped.

In a clinical trial in France, 93 participants who had previously received immune checkpoint blockade and experienced one of these events restarted the therapy. While the same adverse event, or a different one, occurred in 22 patients, the trial indicates that the risk-reward ratio is acceptable if patients are closely monitored.

HS

https://doi.org/10.1038/s41591-019-0528-x

\section{STEM CELLS \\ Maintaining and expanding HSCs}

Cell Stem Cell 25, 1-16 (2019)

Nature https://doi.org/10.1038/s41586-0191244-x (2019)

Hematopoietic stem cells (HSCs) can be maintained and expanded ex vivo for transplantation.

HSC transplantation is a curative therapy for diseases such as leukemia. However, stable HSC culture and expansion has not been reliably achieved, and HSCs are currently cultured in biological products that are risks for infection.

\title{
DIABETES \\ Delaying diabetes onset
}

N. Engl. J. Med. https://doi.org/10.1056/NEJMoa1902226 (2019)

The onset of type I diabetes can be delayed in individuals who are genetically at risk by using an anti-immune therapy.

Type I diabetes is caused by the autoimmune destruction of insulin-producing cells that results in hyperglycemia and the dependence of affected individuals on insulin therapy. Immune interventions aim to prevent the loss of beta cells and progression to full type I diabetes. One such therapy is teplizumab, a monoclonal antibody against CD3.

In a clinical trial of 76 participants who are genetically at risk of developing type I diabetes, those given teplizumab developed type I diabetes on average over 2 years later than those given a placebo. This delay will increase the quality of life of patients with diabetes.

https://doi.org/10.1038/s41591-019-0527-y

Scientists from the United States and Japan collaborated to develop an optimized ex vivo culture system for mouse HSCs that allows expansion of the cells and their subsequent engraftment. Another group in New York was able to maintain human HSCs in vitro by identifying a pathway for HSC maintenance.

Building on this work in the future could mean fewer bone marrow donors will be needed for these transplantations.

HS

https://doi.org/10.1038/s41591-019-0530-3

\section{HUMAN GENETICS}

\section{Widespread tissue genetic mosaics}

Science 364, 970 (2019)

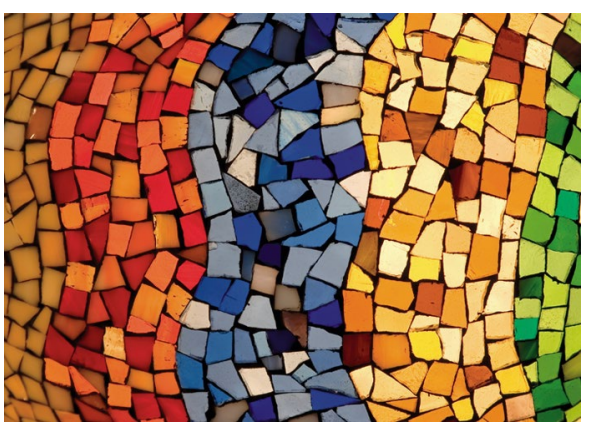

Credit: mihau/E+/ Getty

Healthy adult human tissues are made up of multiple genetic clones.

Mutations in somatic tissues can lead to diseases such as cancer, but the extent to which these mutations occur without incidence of disease is not known.

A group of researchers in the United States developed a pipeline for identifying somatic mutations by comparing the identifiable RNA mutations with those in DNA from the same tissue. By analyzing publicly available data, the authors find that adult tissues are made up of multiple subclones, the number of which varies between tissues.

Factors that affect the number of subclones within the tissues include environmental exposure and cellular division rate. authors also found some of the clones were selected for, suggestive of the early stages of carcinogenesis.

https://doi.org/10.1038/s41591-019-0526-Z

Hannah Stower 